\title{
A facile adenosine triphosphate-responsive nanoplatform for efficacious therapy of esophageal cancer
}

\author{
JINGLONG WANG, LINHAO XU, XIAOTONG LIU, RONGHUA YANG and DONG WANG \\ Department of Thoracic Surgery, Affiliated Hospital of Qingdao University, \\ Qingdao, Shandong 266000, P.R. China
}

Received February 10, 2020; Accepted July 13, 2020

DOI: $10.3892 /$ ol.2020.11969

\begin{abstract}
Current chemotherapeutic agents against esophageal cancer (EC) are suboptimal. To improve treatment efficacy, a nanoplatform based on ATP-responsive drug release was developed for EC therapy. First, the chemotherapeutic agent epirubicin (EPI) was inserted into an ATP aptamer (Ap) to form double-stranded DNA ('DNA duplex'). Subsequently, polyethyleneimine (PEI) was employed to condense the EPI-loaded duplex to construct the final nanoplatform (PEI-Ap-EPI). Following internalization by cancer cells, the EPI-loaded DNA duplex could open and release EPI in an intracellular ATP-rich environment. An in vitro drug-release assay demonstrated that $\sim 50 \%$ of EPI was released from PEI-Ap-EPI in an ATP-rich condition. However, only $15 \%$ of EPI was released in the presence of a low concentration of ATP. In vitro cytotoxicity and apoptosis assays demonstrated that PEI-Ap-EPI could enhance EPI efficiency against EC cells markedly compared with those in the control group. Therefore, this facile PEI-Ap-EPI nanoplatform may be a promising strategy to improve the efficacy of EPI treatment in EC.
\end{abstract}

\section{Introduction}

Esophageal cancer (EC) was the sixth leading cause of cancer-associated mortality worldwide in 2014 (1). Adenocarcinoma and squamous cell carcinoma are the primary types of EC (2). Squamous cell carcinoma is more common in Asia and developing countries (3), and its prognosis is poor (4).

Chemotherapy is a routine method for EC treatment. Commonly used chemotherapeutic agents include platinum drugs, taxanes and epirubicin (EPI) (5). However, low solubility in water and poor selective capability have greatly limited the clinical application of chemotherapeutic agents (6). Therefore,

Correspondence to: Dr Jinglong Wang, Department of Thoracic Surgery, Affiliated Hospital of Qingdao University, 59 Haier Road, Laoshan, Qingdao, Shandong 266000, P.R. China

E-mail: 15063939457@163.com

Key words: esophageal cancer, epirubicin, toxicity, nanoparticles, ATP-responsive it is necessary to develop a novel drug delivery system (DDS) to enhance drug solubility, promote drug accumulation in tumor cells and achieve 'on-demand' drug release, to result in increased efficacy of EC treatment.

Recently, DDSs based on nanoparticles have been developed to deliver antitumor drugs. For example, Fan et al (5) developed EPI-loaded near infrared fluorescent peptide nanoparticles for esophageal cancer therapy; the results revealed that these nanoparticles could significantly enhance the efficiency of EPI and decrease its system toxicity. These 'nano-DDSs' can improve the water solubility, biocompatibility, and tumor-tissue accumulation of a drug via the enhanced permeability and retention effect, and reduce the side effects of a drug (7-9). To achieve on-demand release of a drug, various stimuli-responsive DDSs have been developed. Various endogenous signals, such as $\mathrm{pH}$ and glutathione, have been employed as stimuli to trigger drug release (10). For example, Zhang et al (11) developed a redox-responsive polymeric micelle co-loaded paclitaxel/apatinib for effectively reversing cancer multidrug resistance; the results revealed that in the presence of glutathione, both drugs could rapidly be released to kill cancer cells.

ATP is considered the 'molecular unit of currency' of intracellular energy transfer. ATP exhibits a high concentration in the cytosol of tumor cells $(1-10 \mathrm{mM})$ compared with the extracellular concentration of ATP $(<0.4 \mathrm{mM})$ (12). Therefore, ATP can serve as a stimulus to trigger the release of chemotherapeutic agents.

Aptamers are oligonucleotide/peptide molecules that bind to a specific target molecule (13). Binding of aptamers to ATP has been reported to promote release of preloaded therapeutics directly through a 'conformational switch' that is recognized and activated specifically by ATP (14-16).

Anthracyclines are traditional anticancer drugs. They can destroy cancer cells efficaciously because they interact with the GC pairs of DNA, and inhibit the growth of tumor cells by interfering with DNA transcription (17). Therefore, anthracyclines can be loaded into double-stranded DNA ('DNA duplex')-containing GC pairs.

In the present study, a nano-DDS composed of an ATP aptamer (Ap) and its complementary single-stranded DNA (cDNA), EPI and polyethyleneimine (PEI) was developed. First, the Ap interacted with cDNA to form a duplex by complementation. Subsequently, EPI was loaded into the 
duplex DNA through interaction with the GC pairs in the duplex (Ap-EPI). Finally, PEI (which has a positive charge) underwent electronic interaction with the DNA duplex to condense the DNA duplex into nanoparticles (Fig. 1). It was hypothesized that PEI-Ap-EPI nanoparticles could increase accumulation in tumor cells and release EPI rapidly in the presence of a high level of ATP, thereby improving treatment efficacy considerably.

\section{Materials and methods}

Materials. The Ap (5'-ACCTGGGGGAGTATTGCGGAG GAAGGT-3'), cDNA of the Ap (5'-ACCTTCCTCCGC AATACTCCCCCAGGT-3'), control aptamer (5'-ACCTGG TTTAGGCGGCTCGGGAAT-3') and cDNA of the control aptamer (5'-ATTCCCGAGCCGCCTAAACCAGGT-3') were purchased from Sangon Biotech Co., Ltd. Trypan Blue dye was obtained from Generay Biotech Co., Ltd. RPMI-1640 medium and FBS were purchased from Invitrogen; Thermo Fisher Scientific, Inc. Penicillin was supplied by CSPC Pharmaceutical Group, Ltd. Streptomycin was obtained from Merro Pharmaceutical Co., Ltd. MTT was purchased from Sigma-Aldrich;MerckKGaA.PBS was obtained from Beyotime Institute of Biotechnology. Ethylenediaminetetraacetic acid and $\mathrm{MgCl}_{2}$ were obtained from Sinopharm Chemical Reagent Co., Ltd. Water was purified and deionized using a Milli- $\mathrm{Q}^{\mathrm{TM}}$ system from EMD Millipore.

Cell culture. The EC KYSE-70 and EC109 cell lines were obtained from the American Type Culture Collection, and incubated in RPMI-1640 medium supplemented with $10 \%$ (v/v) FBS, $100 \mathrm{U} / \mathrm{ml}$ penicillin and $100 \mathrm{mg} / \mathrm{ml}$ streptomycin in an atmosphere of $5 \% \mathrm{CO}_{2}$ at $37^{\circ} \mathrm{C}$. Cells were counted using a hemocytometer (Sigma-Aldrich; Merck KGaA). Cell viability was assessed by exclusion of Trypan Blue dye (0.4\%). In brief, $10 \mu \mathrm{l}$ Trypan Blue dye solution was added to $100 \mu \mathrm{l}$ cell suspension, and maintained at room temperature for 3-5 min. Subsequently, $10 \mu \mathrm{l}$ cells suspension was added onto the hemocytometer and observed using a Nikon TS100 light microscope (Nikon Corporation).

DDS preparation. The Ap and its cDNA were dissolved in PBS supplemented with $\mathrm{MgCl}_{2}(5 \mathrm{mM})$, mixed and agitated for $24 \mathrm{~h}$ at room temperature. Subsequently, EPI was added to the DNA duplex and incubated for $2 \mathrm{~h}$ at room temperature to form Ap-EPI. The amount of intercalated EPI was determined based on the fluorescence intensity, which was recorded using a microplate reader (Tecan Group, Ltd.).

Next, PEI-Ap-EPI nanoparticles were created. Briefly, Ap-EPI $(2.5 \mu \mathrm{M})$ was reacted with branched PEI $(25 \mathrm{k})$ at a ratio of 1:2 in aqueous solution for $1 \mathrm{~h}$ at room temperature. Excess PEI was removed by centrifugation at 2,000 $\mathrm{x} g$ for $5 \mathrm{~min}$ at room temperature. As a result, PEI-Ap-EPI was obtained. The final nanoparticles were prepared by resuspending PEI-Ap-EPI in ultrapure water.

The morphology of PEI-Ap-EPI nanoparticles was determined by transmission electron microscopy (TEM) using a Tecnai G2 system (FEI; Thermo Fisher Scientific, Inc.) and their size distribution was determined by differential light scattering (DLS) using a Zs90 setup (Malvern Instruments, Ltd.).
Characterization of Ap-EPI. Ap-EPI $(1.5 \mu \mathrm{M})$ was incubated with $\operatorname{ATP}(0,0.2,0.4,1,2,4$ and $8 \mathrm{mM})$ for $15 \mathrm{~min}$ at $37^{\circ} \mathrm{C}$, and the fluorescence intensity was measured to evaluate the ATP response of Ap-EPI. To evaluate the specificity of the ATP response, Ap-EPI $(2 \mu \mathrm{M})$ was added to PBS supplemented with $\mathrm{MgCl}_{2}(5 \mathrm{mM})$, and incubated with ATP, GTP, uridine triphosphate (UTP) or cytidine triphosphate (CTP), respectively, for $15 \mathrm{~min}$ at $37^{\circ} \mathrm{C}$. All compounds were used at $0,0.5,1,2$, 4 and $8 \mathrm{mM}$, respectively. Finally, fluorescence spectroscopy of EPI was performed at an excitation wavelength of $480 \mathrm{~nm}$ to examine the specificity of Ap-EPI.

ATP-triggered EPI release in vitro. A total of $2 \mathrm{mg}$ of PEI-Ap-EPI (containing $224 \mu \mathrm{g}$ EPI) was dispersed into PBS containing $5 \mathrm{mM} \mathrm{MgCl}$. Subsequently, ATP (0.4 and $4 \mathrm{mM})$ was added, and the mixture was incubated at $37^{\circ} \mathrm{C}$ for $0,0.5,1$, $2,4,8,12$ or $24 \mathrm{~h}$. The amount of EPI released was determined via measurement of fluorescence intensity at the aforementioned time points. In addition, PEI-control (Ctrl) Ap-EPI mixed with ATP (4 mM) was used as a negative control.

Stability of PEI-Ap-EPI nanoparticles. To determine the stability of PEI-Ap-EPI nanoparticles, they were dispersed in PBS with $10 \%$ FBS and incubated at $37^{\circ} \mathrm{C}$. The size distribution and zeta potential of PEI-Ap-EPI nanoparticles were measured using DLS at 0, 2, 4, 6, 8, 12 and $24 \mathrm{~h}$.

EPI accumulation in cells. EPI accumulation in KYSE-70 and EC109 cells was measured by fluorescence microscopy. Cells $\left(\sim 10^{5}\right.$ cells/well $)$ were seeded in a six-well plate. After $24 \mathrm{~h}$, EPI $(0.5 \mu \mathrm{g} / \mathrm{ml})$, Ap-EPI and PEI-Ap-EPI dispersed in serum-free medium were added to the six-well plate, and incubated at $37^{\circ} \mathrm{C}$ for another $24 \mathrm{~h}$. Subsequently, the drugs were removed and cells were washed three times with PBS. Subsequently, cells were incubated with $4 \%$ paraformaldehyde for $15 \mathrm{~min}$ at room temperature. Finally, confocal laser scanning microscopy (CLSM) was performed using an LSM 710 setup (Zeiss AG) after cells had been treated with an anti-fluorescence quencher.

Cell viability assay. KYSE-70 and EC109 cells were seeded into 96-well plates at a density of $5 \times 10^{3}$ cells per well and cultured in medium for $24 \mathrm{~h}$. Then, these two cell lines were treated with increasing concentrations $(0.1,0.5,1,2,4$, 8 and $16 \mu \mathrm{g} / \mathrm{ml}$ EPI for KYSE 70 cells; 0, 0.06, 0.16, 0.32, 0.64, 1.28 and $2.56 \mu \mathrm{g} / \mathrm{ml}$ EPI for EC109 cells; different concentrations were used due to the different sensitivity of the cells to EPI) of free EPI, Ap-EPI or PEI-Ap-EPI, and incubated at $37^{\circ} \mathrm{C}$ for $48 \mathrm{~h}$. Afterwards, these drugs were removed and $20 \mu \mathrm{l} \mathrm{MTT}$ $(5 \mathrm{mg} / \mathrm{ml})$ was added to each well and incubated for another $4 \mathrm{~h}$. Finally, the medium in each well was replaced with $150 \mu \mathrm{l}$ dimethyl sulfoxide. The absorbance was scanned at $490 \mathrm{~nm}$ by FlexStation $^{\mathrm{TM}} 3$ (Molecular Devices, LLC). Each experiment was repeated at least three times. The $\mathrm{IC}_{50}$ value was calculated using GraphPad Prism 6 (GraphPad Software, Inc.).

Apoptosis detection. Apoptosis of KYSE-70 and EC109 cells was detected using the APO-BrdU TUNEL Assay kit (Life Technologies; Thermo Fisher Scientific, Inc.), according to the manufacturer's protocol. These two cell types were seeded 


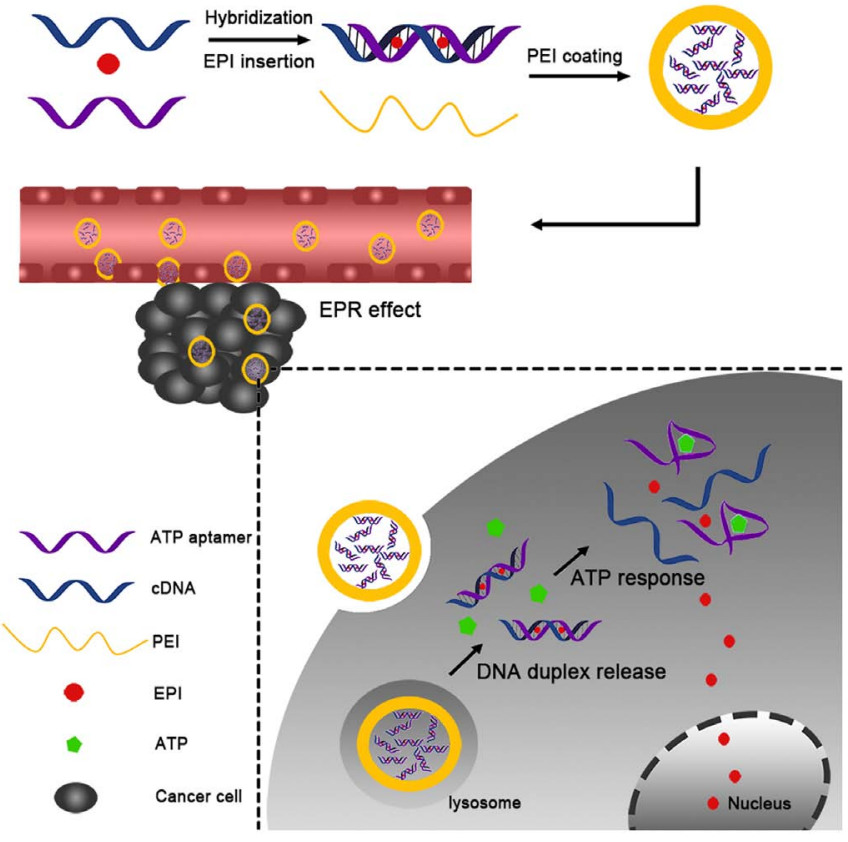

Figure 1. Schematic illustration of PEI-Ap-EPI for enhanced chemotherapy by simultaneous ATP-responsive chemo-drug release and cancer cell sensitization. PEI-Ap-EPI for increased accumulation in tumor tissue through the EPR effect, and effective EPI release in response to the ATP concentration. EPI, epirubicin; EPR, enhanced permeability and retention; PEI, polyethyleneimine.

in six-well plates at a density of $10^{5}$ cells per well. Following culture for $12 \mathrm{~h}$, KYSE-70 cells were incubated with $0.5 \mu \mathrm{g} / \mathrm{ml}$ EPI, Ap-EPI or PEI-Ap-EPI for another $12 \mathrm{~h}$. EC109 cells were treated with the three formulations of EPI $(0.1 \mu \mathrm{g} / \mathrm{ml})$ for $12 \mathrm{~h}$. Subsequent steps were carried out in accordance with the manufacturer's protocol. In brief, cells were stained with BrdU solution $(10 \mu \mathrm{M})$ at $37^{\circ} \mathrm{C}$ for $2 \mathrm{~h}$, washed with PBS for three times, fixed using $4 \%$ formaldehyde at room temperature for 15 min, washed with PBS for three times, incubated with Triton X-100 permeabilization buffer ( $1 \mathrm{ml} /$ well) for $20 \mathrm{~min}$ at room temperature, cultured with $2 \mathrm{~N} \mathrm{HCl}(1 \mathrm{ml})$ for $10 \mathrm{~min}$ at room temperature, incubated with phosphate/citric acid

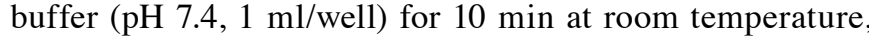
washed with Triton X-100 permeabilization buffer, stained with anti-BrdU primary antibody at room temperature overnight, washed with Triton X-100 permeabilization buffer, and finally stained with FITC-labeled secondary antibody at room temperature for $1 \mathrm{~h}$. Cells were observed under a fluorescence microscope (magnification, x40; Leica Microsystems $\mathrm{GmbH})$. Green fluorescence indicated apoptotic cells. Nuclei were stained with DAPI $(10 \mu \mathrm{g} / \mathrm{ml})$ at room temperature for 15 min and emitted blue fluorescence. Apoptosis of KYSE70 and EC109 cells following treatment with the three drugs was assessed by counting the percentage of apoptotic cells (green) among the total cells (blue) according to the method of Takeda et al (18).

Determination of cell cycle phases. For analyses of cell cycle arrest, KYSE70 and EC109 cells were seeded in a six-well plate at a density of $10^{5}$ cells per well and incubated with EPI, Ap-EPI or PEI-Ap-EPI for $48 \mathrm{~h}$. Then, cells were collected and fixed with $70 \%$ ethanol overnight at $4^{\circ} \mathrm{C}$. Cells were washed with
PBS, exposed to PI/RNase staining buffer (BD Biosciences) and incubated in the dark for $30 \mathrm{~min}$. Cells were counted using a flow cytometer (FACScalibur ${ }^{\mathrm{TM}}$; BD Biosciences) and analyzed using FlowJo 7.6 (FlowJo LLC) (19).

Statistical analysis. Each experiment was repeated three times. Numerical data are presented as the mean \pm SD. One-way single factorial ANOVA followed by Tukey's post hoc test was performed to determine statistical significance of the data using SPSS software (version 19.0; IBM Corp.). P $<0.05$ was considered to indicate a statistically significant difference.

\section{Results}

EPI loading and ATP-response release. First, an Ap and its cDNA were hybridized to construct a carrier to load free EPI. The Ap has 27 base pairs rich in GC that can accommodate EPI (12). To evaluate the number of EPI molecules loaded into the ATP-Duplex, changes in the fluorescence intensity of EPI were measured when it was mixed with various concentrations of the ATP-Duplex. Different concentrations of the ATP-Duplex were added to a fixed concentration of EPI. It was demonstrated that the fluorescence intensity of EPI decreased with an increasing concentration of the ATP-Duplex. Fluorescence nearly disappeared when the molar ratio of duplex: EPI was 1:2 (Fig. 2A).

In the present design, the duplex dissociated once the Ap combined with ATP [which has a different extracellular concentration $(<0.4 \mathrm{mM})$ and cytosol concentration $(1-10 \mathrm{mM})]$, and then EPI was released from the ATP-Duplex. Therefore, to assess the ATP-Duplex response to ATP, the fluorescence intensity of released EPI was recorded after Ap-EPI $(1.5 \mu \mathrm{M})$ had been incubated with ATP $(0,0.2,0.4$, $1,2,4$ or $8 \mathrm{mM})$. In the ATP-Duplex group, the fluorescence intensity strengthened upon a gradual increase in the ATP concentration. When the ATP concentration reached $4 \mathrm{mM}$, the fluorescence intensity of EPI was 2-fold higher than that when the ATP concentration was $0.4 \mathrm{mM}$ (Fig. 2B). These results suggested that the ATP-Duplex was sensitive to ATP, and that EPI release was dependent on the ATP concentration.

To demonstrate the specificity of ATP-triggered EPI release, the release of EPI from Ap-EPI was investigated under GTP, UTP and CTP conditions. Ap-EPI $(2 \mu \mathrm{M})$ was treated with GTP, UTP, CTP or ATP at different concentrations for $12 \mathrm{~h}$, and EPI release was measured using a microplate reader. EPI release was low from Ap-EPI following incubation with GTP, UTP or CTP (Fig. 2C). Furthermore, when the concentration of GTP, UTP and CTP increased to $8 \mathrm{mM}$, $\sim 5 \%$ of fluorescence was recovered. Conversely, fluorescence recovery was $\sim 40 \%$ when $8 \mathrm{mM}$ ATP was added, which was 8 -fold higher than that when GTP, UTP or CTP was added. This result illustrated that the ATP-Duplex response to ATP was specific.

Preparation and characterization of PEI-Ap-EPI. Dizaj et al (20) demonstrated that the DNA formed in nanoparticles can protect them from enzymatic digestion. To avoid the EPI-loaded DNA duplex being digested by enzymes during administration, PEI (a polymer molecule with a positive charge) was employed to condense Ap-EPI 

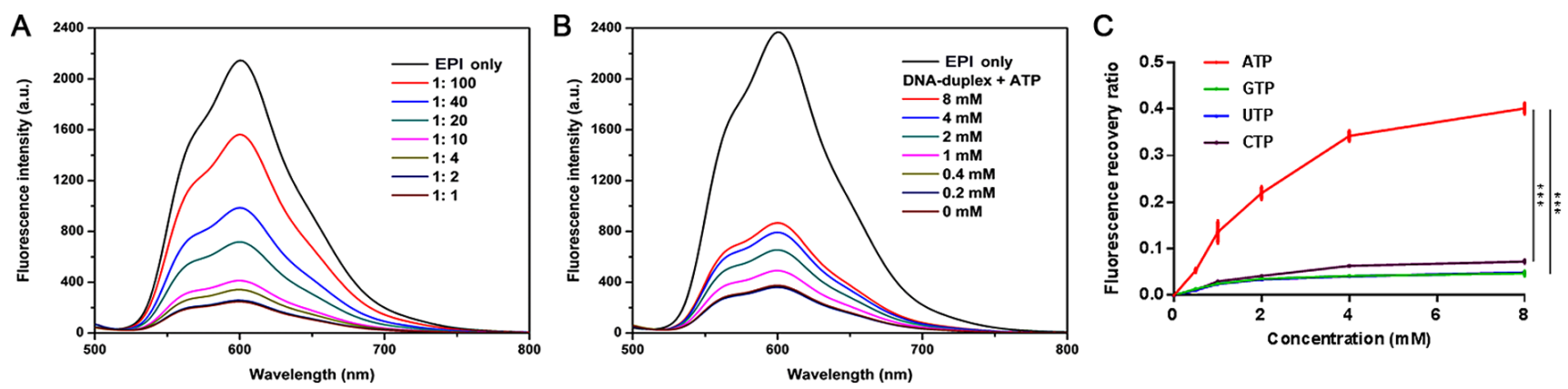

Figure 2. Characterization of Ap-EPI. (A) Fluorescence spectra of EPI solution $(1.5 \mu \mathrm{M})$ with an increasing concentration of the hybridized DNA duplex of the ATP aptamer and its complementary single-stranded DNA following incubation for 15 min in PBS with $\mathrm{MgCl}_{2}$ (5 mM). (B) Fluorescence spectra of Ap-EPI (equivalent to $1.5 \mu \mathrm{M} \mathrm{EPI}$ ) in the presence of ATP $(0,0.2,0.4,1,2,4$ and $8 \mathrm{mM})$ following incubation for 15 min. (C) Fluorescence recovery of Ap-EPI following addition of ATP, GTP, CTP and UTP $(0,0.5,1,2,4$ and $8 \mathrm{mM})$. Error bars indicate the SD $(\mathrm{n}=3) .{ }^{* * *} \mathrm{P}<0.001$. Ap, ATP aptamer; EPI, epirubicin; CTP, cytidine triphosphate; UTP, uridine triphosphate.
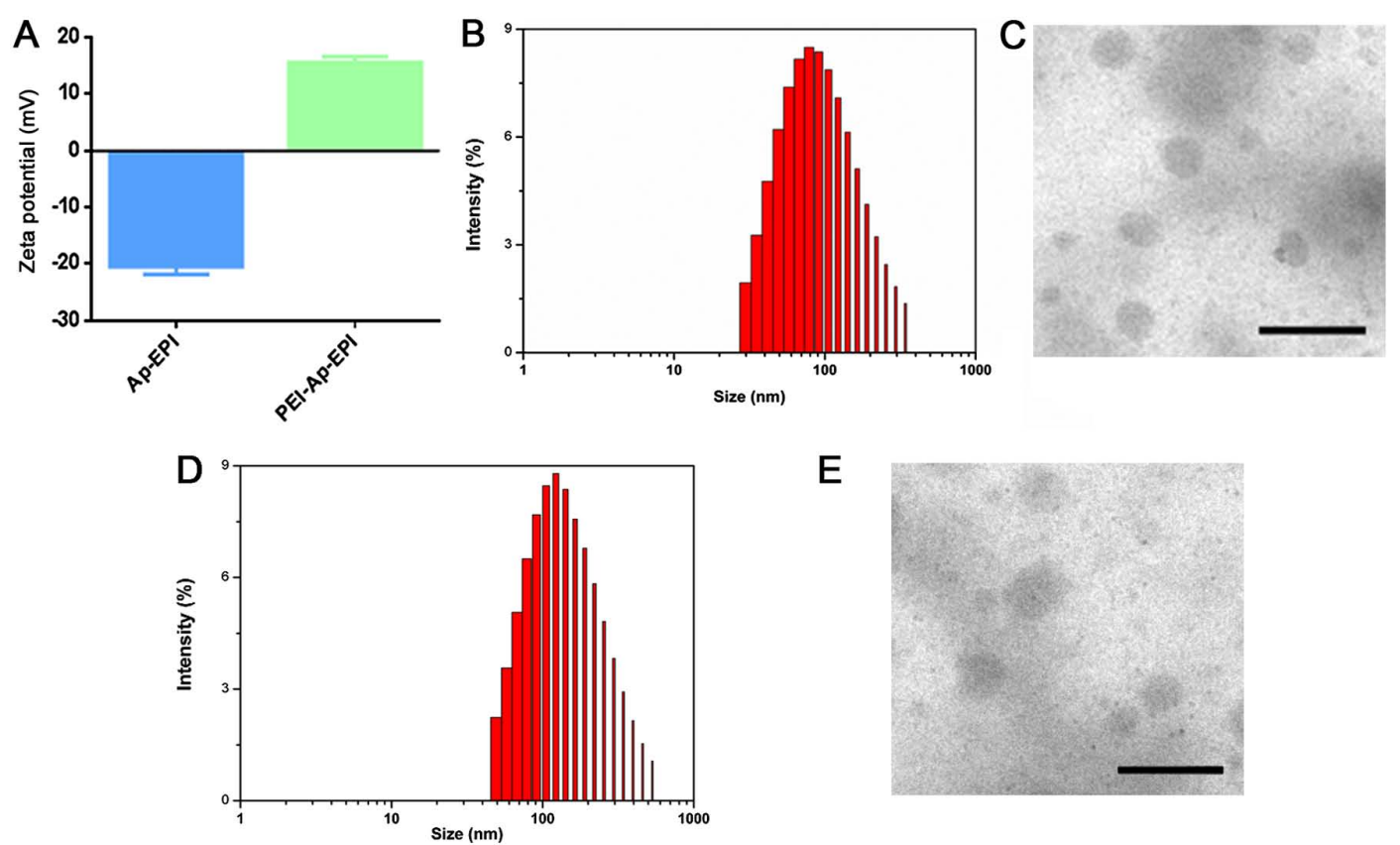

Figure 3. Characterization of PEI-Ap-EPI and PEI-Ctrl-EPI nanoparticles. (A) Surface zeta potential of Ap-EPI and PEI-Ap-EPI. Error bars indicate the SD (n=3). (B) Size distribution of PEI-Ap-EPI. (C) TEM image of PEI-Ap-EPI. Scale bar, $200 \mathrm{~nm}$. (D) Size distribution of PEI-Ctrl-EPI. (E) TEM image of PEI-Ctrl-EPI. Scale bar, $200 \mathrm{~nm}$. Ap, ATP aptamer; Ctrl, control; EPI, epirubicin; PEI, polyethyleneimine; TEM, transmission electron microscopy.

into nanoparticles through charge interaction. To confirm this phenomenon, the zeta-potential changes on the surface of Ap-EPI were measured. The surface charge of Ap-EPI was $-20.4 \mathrm{mV}$, whereas its zeta potential changed to positive $(\sim 15.5 \mathrm{mV})$ after it interacted with PEI (Fig. 3A). This result demonstrated that Ap-EPI interacted completely with PEI. In addition, the size distribution and morphology of PEI-Ap-EPI were examined further by DLS and TEM, respectively. The size of PEI-Ap-EPI measured by DLS was $\sim 100 \mathrm{~nm}$ and the polydispersity index was 0.2 (Fig. 3B), demonstrating that PEI-Ap-EPI was small and of narrow dispersion. In accordance with the results of DLS, TEM also revealed a uniform spheroid structure of size $\sim 100 \mathrm{~nm}$ (Fig. 3C). Additionally, PEI-Ctrl-EPI exhibited a narrow dispersion with uniform spheroid structure (Fig. 3D and E).

Subsequently, to evaluate the ATP-responsivity of PEI-Ap-EPI, the EPI released from PEI-Ap-EPI nanoparticles triggered by ATP was investigated. Typically, ATP of various concentrations was added to PEI-Ap-EPI solutions and incubated for $24 \mathrm{~h}$ at $37^{\circ} \mathrm{C}$. The fluorescence of PEI-Ap-EPI solutions at different time points was recorded to depict the profile of EPI release (Fig. 4A). After $24 \mathrm{~h},>50 \%$ EPI was released from PEI-Ap-EPI in the presence of $4 \mathrm{mM}$ ATP, which was significantly higher compared with the control groups, exhibiting a time-dependent release. In the $0.4 \mathrm{mM}$ ATP group, $\sim 15 \%$ EPI was released. Notably, in the PEI-Ctrl-EPI group, only $\sim 18 \%$ EPI was released after $24 \mathrm{~h}$ in the presence of $4 \mathrm{mM}$ ATP, indicating that PEI-Ctrl-EPI exhibited little response to ATP.

To investigate the stability of PEI-Ap-EPI, the particle size and zeta potential were monitored for $24 \mathrm{~h}$ when it was incubated in PBS with 10\% FBS at pH 7.4. There was little change in particle size and zeta potential, which demonstrated that PEI-Ap-EPI was quite stable in the circulation (Fig. 4B). 

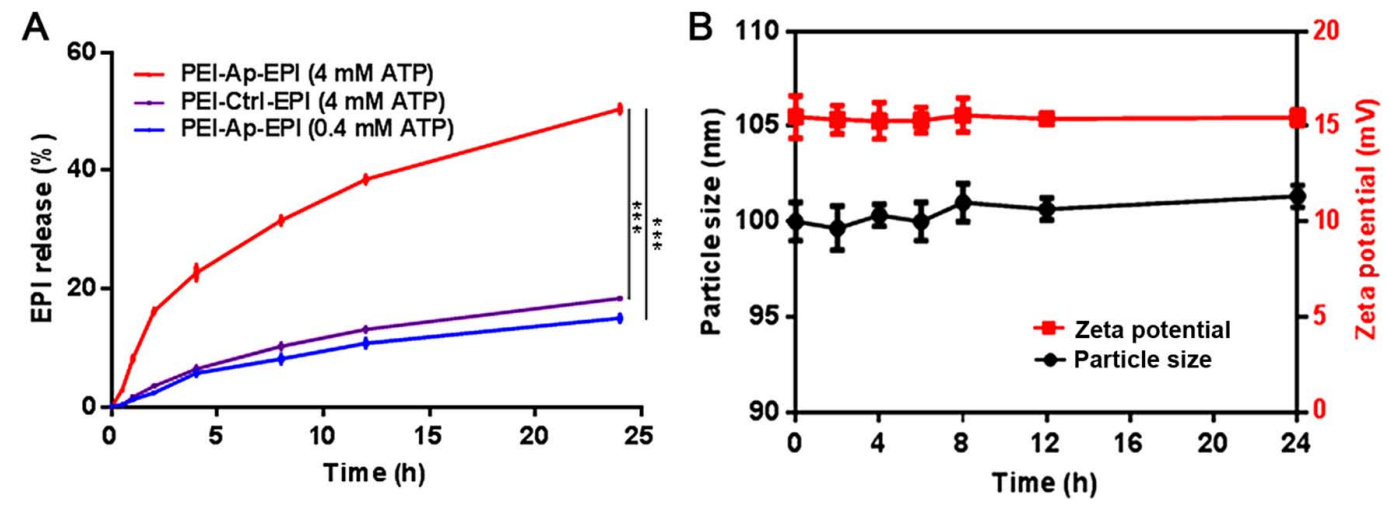

Figure 4. In vitro drug release and stability assay of PEI-Ap-EPI. (A) EPI release from PEI-Ap-EPI with $0.4 \mathrm{mM}$ ATP, PEI-Ap-EPI with $4 \mathrm{mM}$ ATP and PEI-Ctrl-EPI with $4 \mathrm{mM}$ ATP. (B) Measured particle size and zeta potential of PEI-Ap-EPI incubated in PBS (pH 7.4) with $10 \%$ FBS at $37^{\circ} \mathrm{C}$ at various times. Error bars indicate the SD (n=3). ${ }^{* * *} \mathrm{P}<0.001$. Ap, ATP aptamer; Ctrl, control; EPI, epirubicin; PEI, polyethyleneimine.

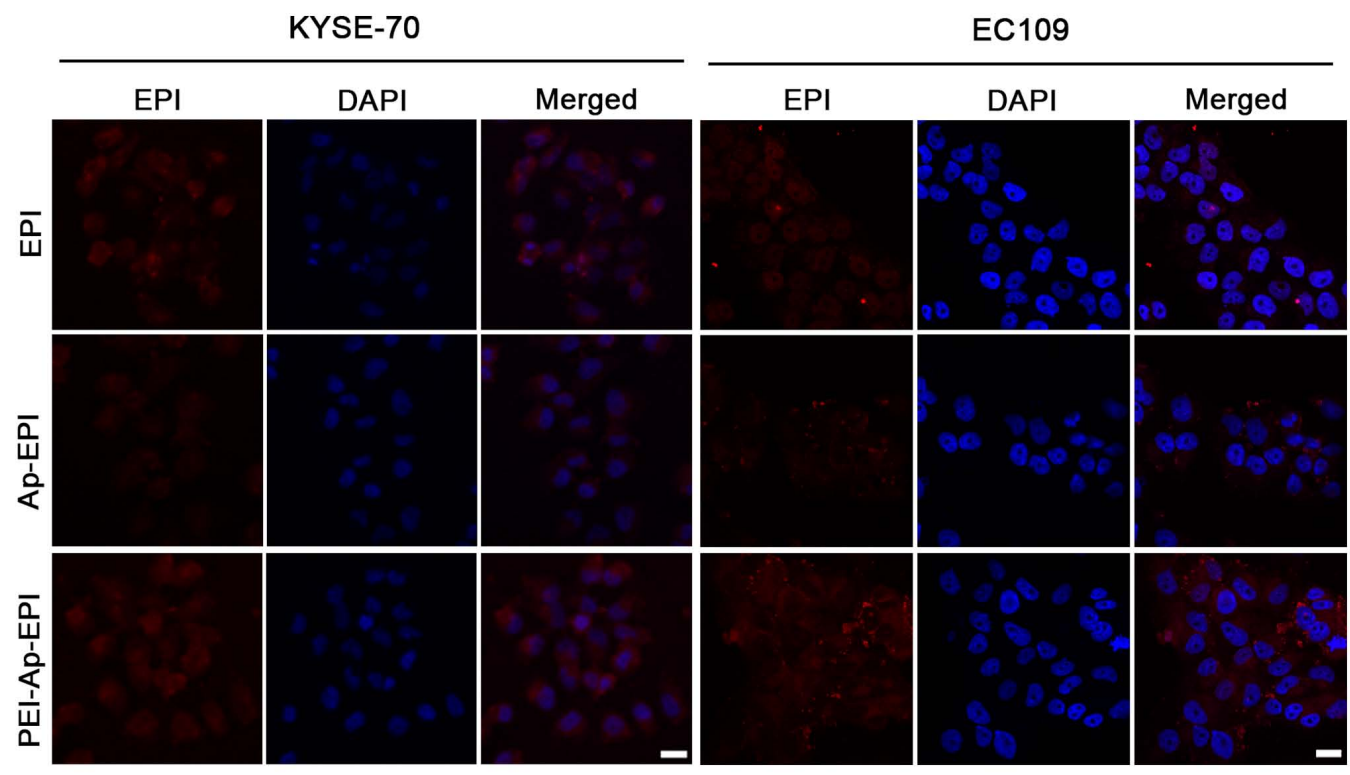

Figure 5. Confocal laser scanning microscopy images of KYSE70 and EC109 cells following treatment with $0.5 \mu \mathrm{g} / \mathrm{ml}$ EPI, Ap-EPI or PEI-Ap-EPI for $24 \mathrm{~h}$. Scale bar, $20 \mathrm{~nm}$. Ap, ATP aptamer; EPI, epirubicin; PEI, polyethyleneimine.

Intracellular accumulation. Sufficient accumulation of a drug in cancer cells is indispensable for efficacious treatment (21). In the present study, intracellular accumulation of EPI, Ap-EPI and PEI-Ap-EPI was measured via fluorescence imaging using CLSM after KYSE-70 and EC109 cells had been treated with these three formulations (Fig. 5). The fluorescence intensity was strongest following treatment with PEI-Ap-EPI, showing the greatest intracellular accumulation. This result could have been due to PEI-Ap-EPI having greater drug loading and being internalized more readily by cancer cells due to the reversal of surface charge after Ap-EPI coating with PEI. Ap-EPI showed low accumulation. The negative surface charge of Ap-EPI likely impeded its internalization.

Cytotoxicity in vitro. The toxicity of EPI, Ap-EPI and PEI-Ap-EPI in KYSE-70 and EC109 cells was evaluated using an MTT assay. The cytotoxicity of the drugs increased with increasing drug dose (Fig. 6). In KYSE-70 cells, the $\mathrm{IC}_{50}$ of PEI-Ap-EPI was $2 \mu \mathrm{g} / \mathrm{ml}$, which was lower than that of EPI
$(2.5 \mu \mathrm{g} / \mathrm{ml})$, but the $\mathrm{IC}_{50}$ of Ap-EPI $(7.5 \mu \mathrm{g} / \mathrm{ml})$ was $\sim 3$ - and 3.7-fold higher than that of EPI and PEI-Ap-EPI, respectively. In EC109 cells, the $\mathrm{IC}_{50}$ of PEI-Ap-EPI was $0.41 \mu \mathrm{g} / \mathrm{ml}$, which was markedly lower than that of EPI and Ap-EPI, and this was consistent with the result in KYSE-70 cells. The reason may be that, without a PEI coating, the surface negative charge of Ap-EPI limits its internalization, impeding its response to extracellular ATP (which is at a low concentration). This result demonstrated that PEI-Ap-EPI had the highest toxicity in KYSE-70 and EC109 cells.

TUNEL assay. The TUNEL assay was utilized to demonstrate the DNA damage that PEI-Ap-EPI can elicit. After KYSE-70 and EC109 cells were incubated with EPI, Ap-EPI or PEI-Ap-EPI, a TUNEL assay was performed and fluorescence microscopy was used to capture images. Blue fluorescence indicated nuclei whereas green fluorescence indicated apoptotic cells (Fig. 7A). In the PEI-Ap-EPI group, more green fluorescence was observed, indicating that it 

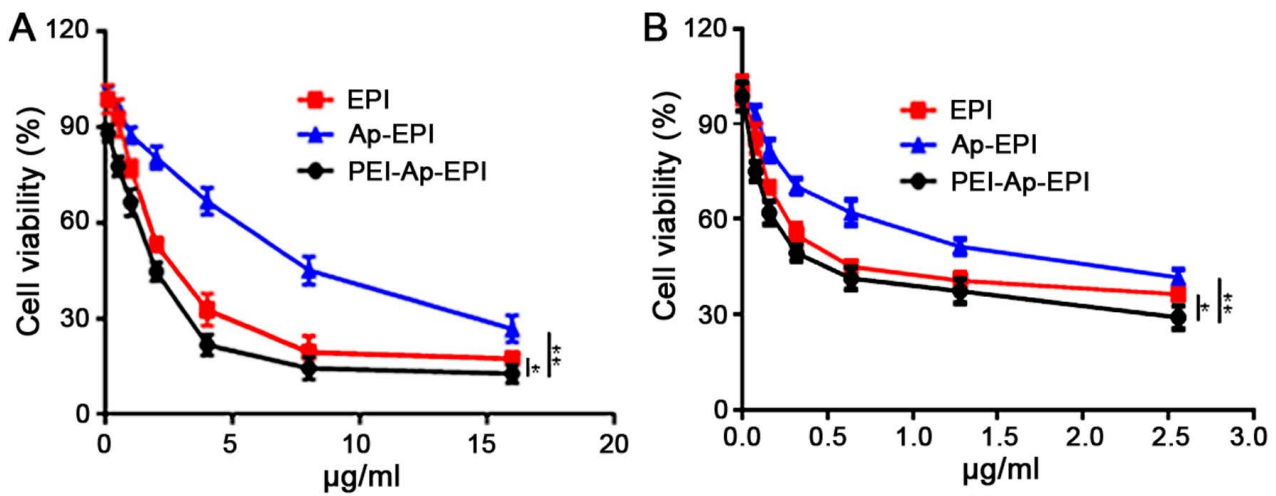

Figure 6. In vitro toxicity of EPI, Ap-EPI and PEI-Ap-EPI on (A) KYSE70 and (B) EC109 cells. Error bars indicate the $\mathrm{SD}(\mathrm{n}=3) .{ }^{*} \mathrm{P}<0.05 ;{ }^{* *} \mathrm{P}<0.01$. Ap, ATP aptamer; EPI, epirubicin; PEI, polyethyleneimine.
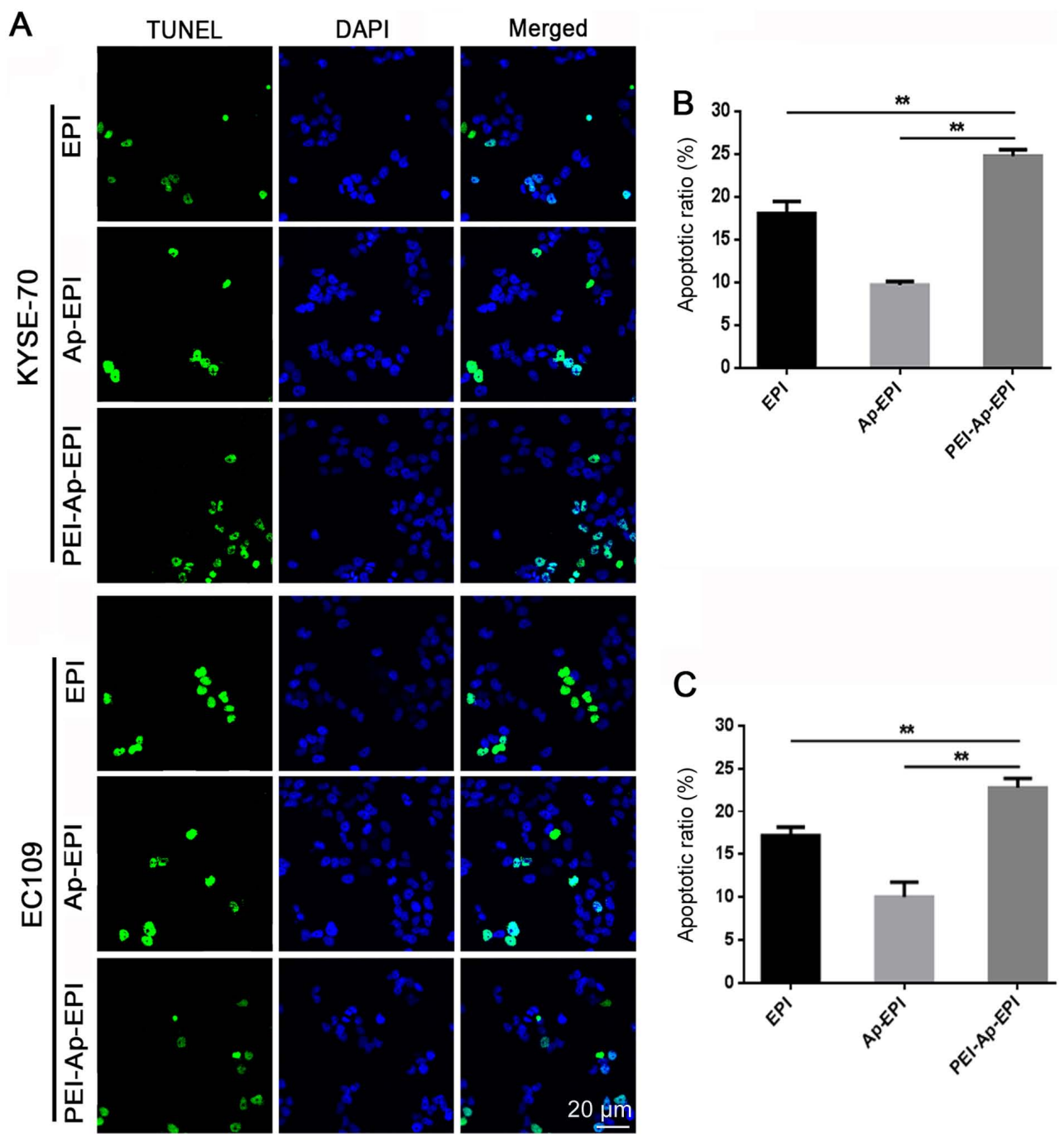

Figure 7. TUNEL analysis. (A) Images of the TUNEL assay acquired using a fluorescence microscope. Green fluorescence represents apoptotic cells. Blue fluorescence denotes nuclei. Scale bar, $20 \mu \mathrm{m}$. (B) Apoptotic ratio of KYSE70 cells following treatment with $0.5 \mu \mathrm{g} / \mathrm{ml}$ EPI, Ap-EPI or PEI-Ap-EPI for $12 \mathrm{~h}$. (C) Apoptotic ratio of EC109 cells following treatment with $0.1 \mu \mathrm{g} / \mathrm{ml}$ EPI, Ap-EPI or PEI-Ap-EPI for $12 \mathrm{~h}$. Error bars indicate the SD (n=3). ${ }^{* *} \mathrm{P}<0.01$. Ap, ATP aptamer; EPI, epirubicin; PEI, polyethyleneimine.

caused more severe damage than the other two groups. After counting the number of apoptotic cells in the three groups, it was demonstrated that PEI-Ap-EPI had the strongest ability to cause DNA damage. The apoptotic index (AI) in
KYSE-70 cells was $\sim 25 \%$, which was higher than that of EPI $(18 \% ; \mathrm{P}=0.008)$ and Ap-EPI $(10 \% ; \mathrm{P}=0.005)$, respectively (Fig. 7B). The AI of PEI-Ap-PEI in EC109 cells was similar to that in KYSE cells $(\sim 23 \%)$ and the AI of EPI and Ap-EPI 

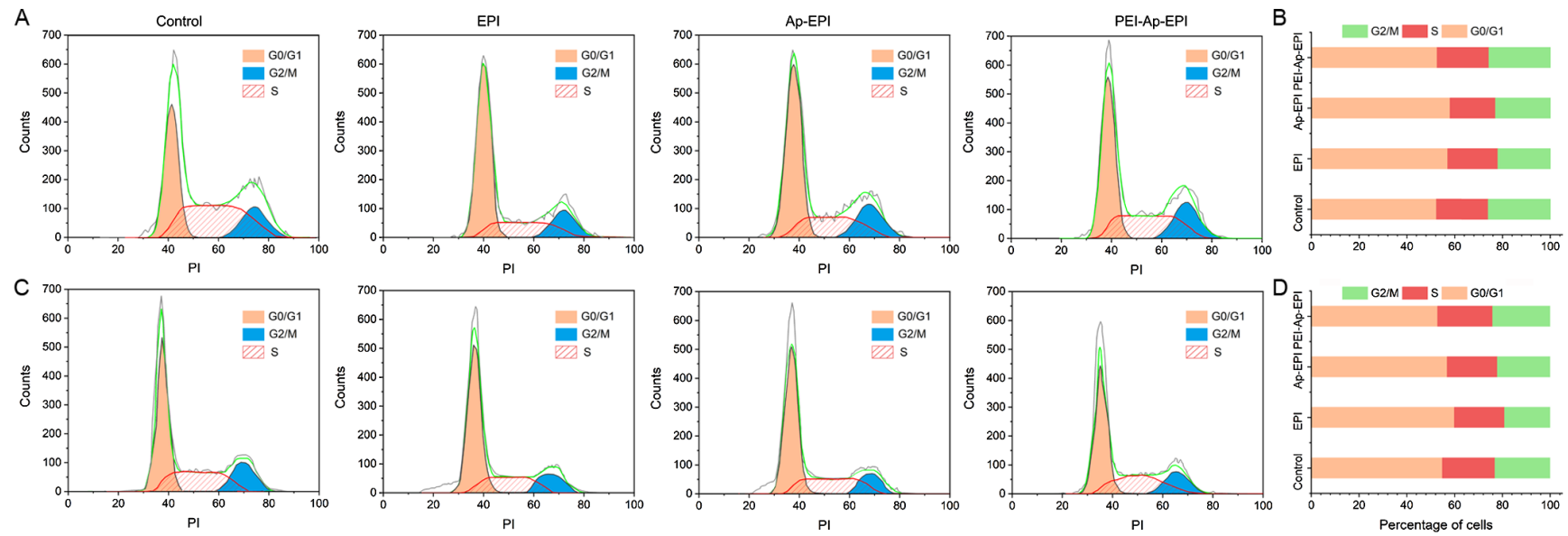

Figure 8. Proportion of cells in each cell cycle phase after (A and B) KYSE-70 and (C and D) EC109 cells were treated with different formulations of EPI. Ap, ATP aptamer; EPI, epirubicin; PEI, polyethyleneimine.

was $17 \%(\mathrm{P}=0.007)$ and $10 \%(\mathrm{P}=0.004)$, respectively (Fig. 7C).

Cell cycle arrest. To investigate the influence of different formulations of EPI on cell-cycle arrest, cells at each phase were counted by flow cytometry (Fig. 8). In KYSE70 and EC109 cells, after they had been treated with the three formulations of EPI, the proportion of cells in the $G_{0} / G_{1}$ phase was the greatest, and the cell cycle distribution did not differ among the three formulations.

\section{Discussion}

Chemotherapy is a conventional way to inhibit tumor growth. However, some disadvantages, such as poor selectivity, weaken the efficacy of chemotherapy. Therefore, overcoming the shortcomings of chemotherapeutics to improve the efficacy of EC therapy would be helpful, and using nanoparticles for drug delivery may be an effective method.

ATP is considered to be the 'molecular unit of currency' of intracellular energy transfer. ATP is present at high concentrations in the cytosol of tumor cells (1-10 mM) compared with the extracellular concentration of ATP $(<0.4 \mathrm{mM})(12)$. The distinct difference in the ATP levels between the extracellular and intracellular milieu is the biological principle for the design of ATP-responsive carriers. In the present study, an ATP-responsive nanoplatform for EPI delivery for EC treatment was developed. It was demonstrated that an Ap composed of 27 base pairs rich in GC accommodated EPI following interaction with its cDNA. The in vitro drug release experiments demonstrated that EPI can be rapidly released in the presence of intracellular ATP $(4 \mathrm{mM})$, but only a small amount of EPI is released in the presence of extracellular ATP $(0.4 \mathrm{mM})$. These actions contributed to high drug loading and release of the active drug in cells, resulting in toxicity to tumor cells.

PEI was selected to condense the DNA duplex due to four main reasons. First, through the charge interaction between PEI and DNA duplex, nanoparticles can be formed, which contribute to drug accumulation in tumor tissues (22). Second, condensation with PEI can overcome DNA instability in vivo and reduce premature leakage of EPI (12). Third, PEI can become protonated in the acid environment of lysosomes, resulting in disassembly of PEI-Ap-EPI nanoparticles and promotion of DNA-duplex escape from lysosomes (23), which, ultimately, helps to enhance therapy efficacy. Fourth, the DNA duplex can have a negative charge, thereby impeding its internalization by cancer cells (24). Following interaction with PEI, the charge of the obtained nanoparticles changed to positive, which was conducive to cell internalization.

KYSE-70 and EC109 cells were used to investigate the efficacy of PEI-Ap-EPI. It was demonstrated that more EPI accumulated in cells after they had been treated with PEI-Ap-EPI. A possible reason is that PEI-Ap-EPI has more drug-loading sites and is internalized more readily by cancer cells due to surface-charge reversal after Ap-EPI coating with PEI. The group treated with Ap-EPI exhibited little accumulation of EPI. The negative surface charge of Ap-EPI likely impeded its internalization.

In in vitro cytotoxicity experiments, the low $\mathrm{IC}_{50}$ of PEI-Ap-EPI could be explained by relatively high drug loading and effective internalization. In accordance with this hypothesis, absence of PEI coating resulted in poor internalization, which contributed to the high $\mathrm{IC}_{50}$. The TUNEL assay result was consistent with the data from experiments on intracellular accumulation and the MTT assay. These results suggested that PEI-Ap-EPI had improved efficacy for EC cells. In addition, the present study investigated the influence of drug formulations on cell cycle arrest and but did not find a significant difference among them.

The main limitation of the present study was the lack of in vivo experiments. Clinical application of the nano-DDS can occur only after rigorous in vivo experiments have been completed.

In conclusion, a novel DDS (PEI-Ap-EPI nanoparticles) for EC treatment was constructed. PEI-Ap-EPI nanoparticles of size $\sim 100 \mathrm{~nm}$ were responsive specifically to a high concentration of ATP in EC cells, and were stable in the presence of FBS. In vitro experiments demonstrated that PEI-Ap-EPI could increase EPI accumulation in tumor cells. PEI-Ap-EPI showed higher cytotoxicity, and caused more severe DNA damage than Ap-EPI and EPI. These results illustrated that the novel nano-DDS may be efficacious in EC treatment, and has higher efficacy than EPI alone. 


\section{Acknowledgements}

Not applicable.

\section{Funding}

The present study was supported by the Qingdao University Development and Innovation Found (grant no. 201825QUDIF124).

\section{Availability of data and materials}

The datasets used and/or analyzed during the present study are available from the corresponding author on reasonable request.

\section{Authors' contributions}

JW and LX conceived the study, and analyzed and interpreted the data. XL performed the experiments, analyzed data and wrote the manuscript. RY and DW performed the experiments and analyzed data. JW and DW designed experiments, analyzed the data, polished the manuscript and guided the reply to the comments. All authors read and approved the final manuscript.

\section{Ethics approval and consent to participate}

Not applicable.

\section{Patient consent for publication}

Not applicable.

\section{Competing interests}

The authors declare that they have no competing interests.

\section{References}

1. Pakzad R, Mohammadian-Hafshejani A, Khosravi B Soltani S, Pakzad I, Mohammadian M, Salehiniya H and Momenimovahed Z: The incidence and mortality of esophageal cancer and their relationship to development in Asia. Ann Trans Med 4: 29, 2016

2. Bandla S, Pennathur A, Luketich JD, Beer DG, Lin L, Bass AJ, Godfrey TE and Litle VR: Comparative genomics of esophageal adenocarcinoma and squamous cell carcinoma. Ann of Thorac Surg 93: 1101-1106, 2012.

3. Zhang HZ, Jin GF and Shen HB: Epidemiologic differences in esophageal cancer between Asian and Western populations. Chin J Cancer 31: 281-286, 2012.

4. Crane SJ, Locke GR III, Harmsen WS, Zinsmeister AR, Romero Y and Talley NJ: Survival trends in patients with gastric and esophageal adenocarcinomas: A population-based study. Mayo Clin Proc 83: 1087-1094, 2008.

5. Fan Z, Chang Y, Cui C, Sun L, Wang DH, Pan Z and Zhang M Near infrared fluorescent peptide nanoparticles for enhancing esophageal cancer therapeutic efficacy. Nat Commun 9: 2605 , 2018.
6. Liu Y, Zhang B and Yan B: Enabling anticancer therapeutics by nanoparticle carriers: The delivery of Paclitaxel. Int J Mol Sci 12: 4395-4413, 2011.

7. Wen $\mathrm{H}$, Jung $\mathrm{H}$ and Li X: Drug delivery approaches in addressing clinical pharmacology-related issues: Opportunities and challenges. Aaps J 17: 1327-1340, 2015.

8. Greish K: Enhanced permeability and retention (EPR) effect for anticancer nanomedicine drug targeting. Methods Mol Biol 624: 25-37, 2010.

9. PatraJK,Das G,FracetoLF,Campos EVR,Rodriguez-TorresMDP, Acosta-Torres LS, Diaz-Torres LA, Grillo R, Swamy MK, Sharma S, et al: Nano based drug delivery systems: Recent developments and future prospects. J Nanobiotechnology 16: 71, 2018.

10. Mo R and Gu Z: Tumor microenvironment and intracellular signal-activated nanomaterials for anticancer drug delivery. Mater Today 19: 274-283, 2016.

11. Zhang X, Ren X, Tang J, Wang J,Zhang X, He P, YaoC, Bian W and Sun L: Hyaluronic acid reduction-sensitive polymeric micelles achieving co-delivery of tumor-targeting paclitaxel/apatinib effectively reverse cancer multidrug resistance. Drug Deliv 27: 825-835, 2020

12. Mo R, Jiang T, DiSanto R, Tai W and Gu Z: ATP-triggered anticancer drug delivery. Nat Commun 5: 3364, 2014.

13. Tan Y, Shi YS, Wu XD, Liang HY, Gao YB, Li SJ, Zhang XM, Wang F and Gao TM: DNA aptamers that target human glioblastoma multiforme cells overexpressing epidermal growth factor receptor variant III in vitro. Acta Pharmacol Sin 34: 1491-1498, 2013.

14. Huizenga DE and Szostak JW: A DNA aptamer that binds adenosine and ATP. Biochemistry 34: 656-665, 1995.

15. Modh H, Witt M, Urmann K, Lavrentieva A, Segal E, Scheper T and Walter JG: Aptamer-based detection of adenosine triphosphate via qPCR. Talanta 172: 199-205, 2017.

16. Heilkenbrinker A, Reinemann C, Stoltenburg R, Walte JG, Jochums A, Stahl F, Zimmermann S, Strehlitz B and Scheper T: Identification of the target binding site of ethanolamine-binding aptamers and its exploitation for ethanolamine detection. Anal Chem 87: 677-685, 2015.

17. Canzoneri JC and Oyelere AK: Interaction of anthracyclines with iron responsive element mRNAs. Nucleic Acids Res 36: 6825-6834, 2008.

18. Takeda K, Uchiyama K, Kinukawa M, Tagami T, Kaneda M and Watanabe S: Evaluation of sperm DNA damage in bulls by TUNEL assay as a parameter of semen quality. J Reprod Dev 61: 185-190, 2015

19. Guo JR, Chen QQ, Lam CW and Zhang W: Effects of karanjin on cell cycle arrest and apoptosis in human A549, HepG2 and HL-60 cancer cells. Biol Res 48: 40, 2015.

20. Dizaj SM, Jafari S and Khosroushahi AY: A sight on the current nanoparticle-based gene delivery vectors. Nanoscale Res Lett 9: 252, 2014

21. Wang L, Lin X, Wang J, Hu Z, Ji Y, Hou S, Zhao Y, Wu X and Chen C: Novel insights into combating cancer chemotherapy resistance using a plasmonic nanocarrier: Enhancing drug sensitiveness and accumulation simultaneously with localized mild photothermal stimulus of femtosecond pulsed laser. Adv Funct Mater 24: 4229-4239, 2014.

22. Blanco E, Shen H and Ferrari M: Principles of nanoparticle design for overcoming biological barriers to drug delivery. Nat Biotechnol 33: 941-951, 2015 .

23. Akinc A, Thomas M, Klibanov AM and Langer R: Exploring polyethylenimine-mediated DNA transfection and the proton sponge hypothesis. J Gene Med 7: 657-663, 2005.

24. Liu Y, Xu CF, Iqbal S, Yang XZ and Wang J: Responsive nanocarriers as an emerging platform for cascaded delivery of Nucleic acids to cancer. Adv Drug Deliv Rev 115: 98-114, 2017.

This work is licensed under a Creative Commons Attribution-NonCommercial-NoDerivatives 4.0 International (CC BY-NC-ND 4.0) License. 